Research Paper

\title{
Monitoring Wildlife and their Habitats in the Southern Ocean and Around Indian Research Stations in Antarctica
}

\author{
ANANT PANDE, K SIVAKUMAR, S SATHYAKUMAR*, R SURESH KUMAR, J A JOHNSON, SAMRAT \\ MONDOL and VINOD B MATHUR \\ Wildlife Institute of India, Chandrabani, Dehradun, Uttarakhand 248 001, India
}

(Received on 15 June 2016; Accepted on 13 December 2016)

\begin{abstract}
Antarctica and its surrounding ocean are at the centre stage of rapid and extreme environmental events due to prevailing global climate change, which in turn necessitates long-term monitoring of wildlife and their habitats in this region. Systematic monitoring of seabirds and marine mammals were carried out during five austral summers (2008-09, 2009-10, 2013-14, 2014-15 and 2015-16). Vessel-based surveys for pelagic seabirds in the southern Indian Ocean, aerial surveys for seals and penguins and field surveys for locating nesting sites of Antarctic birds were conducted at Indian area of operation in Antarctica. Forty-nine species of seabirds were observed during the vessel-based surveys. Species richness peaked in the mid-latitudes but species abundances increased along higher latitudes towards Antarctica. Planktivorous species in the lower latitudes were replaced by mixed prey dependent species in the higher latitudes. Aerial surveys recorded a total of four species of seals viz. Weddell seal, Crabeater seal, Leopard seal and Ross seal and two penguin species viz. Emperor penguin and Adelie penguin. Out of 15 islands in Larsemann hills that were surveyed on-foot, the presence of nesting sites of seabirds is reported from 13 islands. The preliminary results from our study provide the baseline data for ecologically important species within Indian area of operation in Antarctica and will help design future research activities.
\end{abstract}

Keywords: Larsemann Hills; Schirmacher Oasis; Prydz Bay; India Bay; Aerial Survey; Nest Monitoring

\section{Introduction}

Global warming has been identified as a major driver of change in the Antarctic ecosystem, some areas in the continent warming more rapidly than other parts of the world (Hansen et al., 1999). Though, there are marked variations in the responses of the Antarctic terrestrial and marine communities to the annual climatic variations (Walther et al., 2002), more information is needed on understanding the sensitivity of key ecological species to primary biological and physical driving forces in the continent. These temporal variations in the Antarctic environment are considered to play a major role in the primary production, benthic recruitment rates and vertebrate population dynamics. Modifications in the cold climate of southern ocean and Antarctica will affect the community composition of primary producers, thereby affecting the higher trophic levels (Croxall et al., 2002; Agusti et al., 2010; Constable et al., 2014).

\footnotetext{
*Author for Correspondence: E-mail: ssk@wii.gov.in
}

Marine vertebrates, being ecologically important and threatened with climatic change (Sydeman et al., 2015), have long been identified as ecosystem sentinels. They are influenced by oceanographic processes which play a large role in determining their abundance and distribution (Abrams 1985; Bost et al., 2009; Ribic et al., 2011; Commins et al., 2013). However, population trends are known for only a few marine mammal species (Constable et al., 2014), and for seabirds the effects of climatic change have not been studied in depth for most of the species. Seabird species from southern ocean such as penguins have been demonstrated to respond dramatically to seaice variations over the past century (Smith et al., 1999; Ainley et al., 2003). However, the underlying processes linking climate variation to these top predators remains unclear (Sydeman et al., 2015; Trathan et al., 2015). 
Long-term monitoring of seabird and marine mammal populations is thus needed to provide crucial information about any kind of fluctuations over a period of time. Conventions in the Antarctic treaty, i.e., Convention on the Conservation of Antarctic Seals (CCAS) and the Convention for the Conservation of Marine Living Resources (CCAMLR) also stress the importance of ecosystem monitoring through such species.

In this context, the Wildlife Institute of India (WII), Dehradun, has been monitoring seabirds, mammals and their habitats in the southern ocean and around the Indian Research Stations in Antarctica since the early 1990s (Sathyakumar 1995; Bhatnagar and Sathyakumar 1997; Hussain and Saxena, 2008).

WII initiated Phase-I of the monitoring program titled "Long-term monitoring of wildlife and habitats in Antarctica and Southern Ocean" from 2008-10 (Sivakumar and Sathyakumar 2012; Kumar and Johnson, 2014) followed by a phase-II from 20132016 (Pande et al., 2014). This program was undertaken to ascertain the status of key species of the southern ocean and Antarctica and establish a protocol for long-term monitoring of key indicator species around Indian research stations. The present study presents a preliminary assessment of the data compiled during these past five Indian Scientific Expeditions to Antarctica (InSEA).

\section{Study Area}

\section{Southern Indian Ocean (African Sector) and Southern Ocean}

The voyage route of Indian Scientific Expedition to Antarctica (InSEA) from Cape Town, South Africa (S 33 55' 25.59" E $18^{\circ} 25^{\prime} 24.04^{\prime \prime}$ ) to Larsemann hills, east Antarctica (68 $\left.{ }^{\circ} 54^{\prime} 92.1^{\prime \prime} \mathrm{S}, 7^{\circ} 30^{\prime} 40.2^{\prime \prime} \mathrm{E}\right)$ to Indian barrier (India Bay), Princess Astrid Coast (S $70^{\circ} 7^{\prime} 47.34^{\prime \prime}$ E $\left.12^{\circ} 23^{\prime} 51.9^{\prime \prime}\right)$ and back to Cape Town (Fig. 1) was used for seabird and marine mammal monitoring. The voyage covered a latitudinal stretch of southern Indian Ocean from $38^{\circ} \mathrm{S}$ to $60^{\circ} \mathrm{S}$ and southern ocean from $60^{\circ} \mathrm{S}$ to $70^{\circ} \mathrm{S}$. The longitudes covered were from $9^{\circ} 37^{\prime} \mathrm{E}$ to $76^{\circ} 50^{\prime} \mathrm{E}$.

Larsemann Hills, Prydz Bay, Antarctica : Larsemann hills $\left(69^{\circ} 20^{\prime} \mathrm{S}\right.$ to $69^{\circ} 30^{\prime} \mathrm{S}$ Latitude; $75^{\circ}$ $55^{\prime} \mathrm{E}$ to $76^{\circ} 30^{\prime} \mathrm{E}$ Longitude), East Antarctica, are a group of islands at Prydz Bay (Fig. 2). It is an icefree oasis on the Ingrid Christensen Coast, Princess Elizabeth Land, located approximately midway between the eastern extremity of the Amery Ice Shelf and the southern boundary of the Vestfold Hills, flanked on both sides by two large peninsulas, the western Stornes and the eastern Broknes, which enclose a group of variously sized islands and peninsulas. Together the islands form the second largest group of four major ice-free oases found along East Antarctica's $5000 \mathrm{~km}$ long coastline spread over an area of about $50 \mathrm{~km}^{2}$ (Hodgson et al., 2009). India's $3^{\text {rd }}$ permanent research station in Antarctica, BHARATI, is located on Grovnes peninsula. Four other Antarctic stations, viz. the Progress I \& Progress II (Russia), Law- Racovita (Australia-Romania) and Zhongshan (China) are located along the edge of the Broknes peninsula.

\section{Schirmacher Oasis, Central Dronning Maudland}

The Schirmacher Oasis is situated on the Princess Astrid coast of Dronning Maud Land, Antarctica between the Fimbul ice shelf and continental icecap (Fig. 3). This ice-free land is spread across an area of about $34 \mathrm{~km}^{2}$ between the coordinates $70^{\circ} 46^{\prime} 04^{\prime \prime}$ 44' 21" S and 11'49' 54"-26' 03" E (Singh et al., 2012). Second permanent Indian research station MAITRI is located on the south-eastern part of the oasis.

\section{India Bay, Princess Astrid Coast}

India Bay, as it is called by the Indian expeditioners, is part of the Haakon VII sea next to the Fimbul ice shelf where Indian expedition activities are conducted $\left(69^{\circ} 47^{\prime}-69^{\circ} 48^{\prime} \mathrm{S}\right.$ and $10^{\circ} 33^{\prime}-12^{\circ} 58^{\prime} \mathrm{E}$ (Fig. 4).

\section{Methods}

\section{Vessel-based Surveys}

We conducted vessel-based surveys on the voyage route of Indian expedition vessels in the Southern Indian Ocean and Southern Ocean. These surveys were conducted onboard ice-class vessels chartered by the ESSO - National Centre of Antarctic \& Ocean Research (NCAOR), Ministry of Earth Sciences, Government of India onboard MV Emerald Sea (200809; $28^{\text {th }}$ InSEA) and M V Ivan Papanin (2009-10, $2013-14,2014-15 \& 2015-16 ; 29^{\text {th }}, 33^{\text {rd }}, 34^{\text {th }} \& 35^{\text {th }}$ 

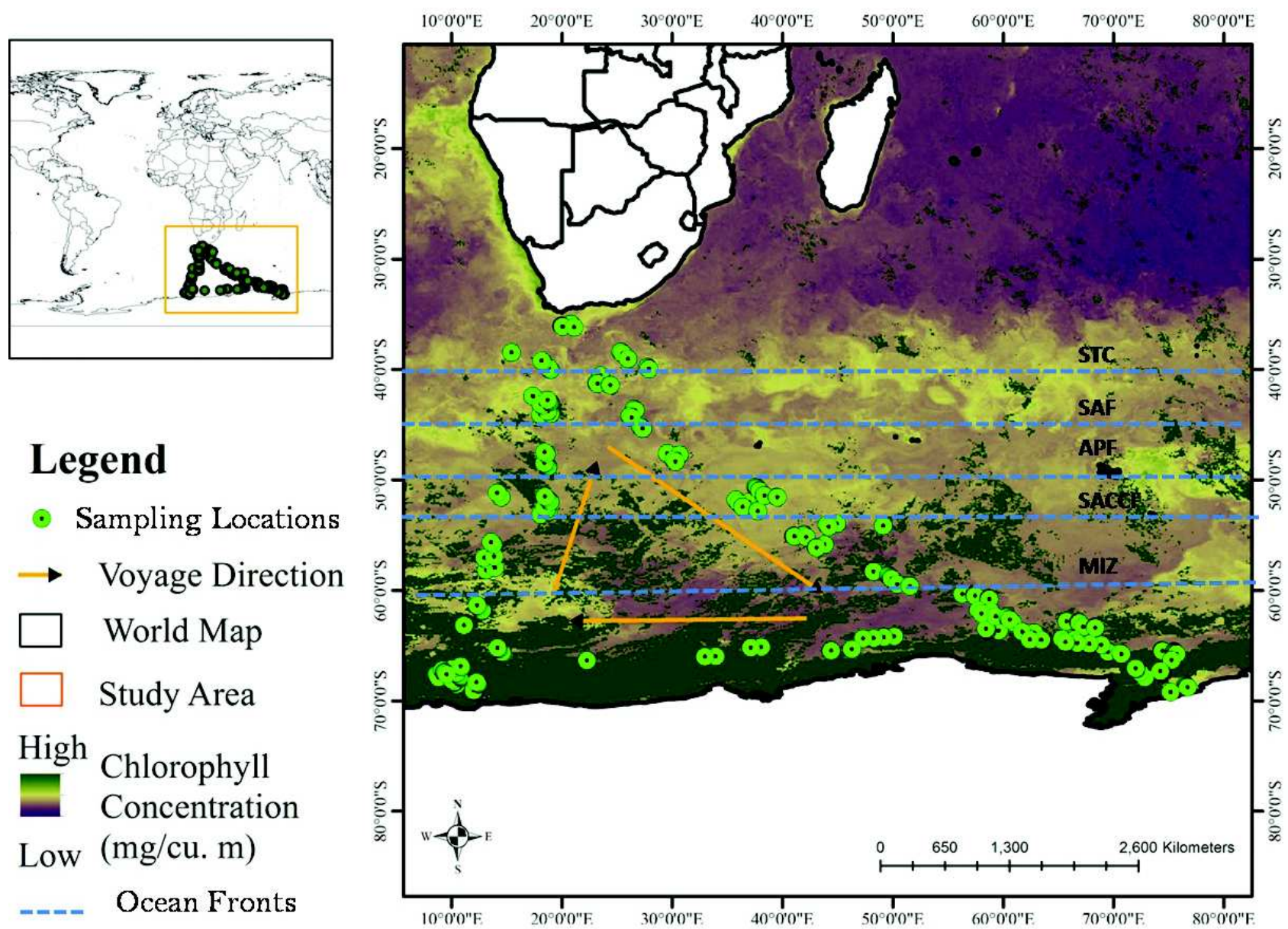

Fig. 1: Sampling route for the vessel-based surveys in the Indian Scientific Expedition to Antarzctica in the Southern Indian Ocean. STC - Sub-tropical Current, SAF - Sub-Antarctic Front, APF - Antarctic Polar Front, SACCF - Southern Antarctic Circumpolar Current Front, MIZ - Marginal Ice Zone (front abbreviations from Commins et al. 2013). Average chlorophyll values of December 2013 taken from NASA Earth Observations http://neo.sci.gsfc.nasa.gov/

InSEA respectively). Observations for oceanic birds and marine mammals were carried out from the bridge of these vessels (approx. height from sea level $\sim 40$ $\mathrm{m})$ in appropriate weather days to estimate their abundance and distribution pattern along the voyage route.

All the seabirds, flying across the bow of the ship, up to $300 \mathrm{~m}$ from the ship or visible to naked eye, were counted during the daylight hours (Tasker et al., 1984) and a visual estimation was made of their perpendicular distance from the ship route. Only those birds were counted which could be seen without any ocular aid, although, once sighted the identity was confirmed with the help of binoculars. Birds which were foraging together be within 2-5 $\mathrm{m}$ of each other were considered to be as one cluster. Marine mammals seen from the bridge of the ship were identified based on their spout pattern, dorsal fin shape, diving pattern and tail fluke shape with the help of binoculars.

Observations were also made during the ship's movement through pack ice, when the cruising speed was at least 6 Knots or more. Observations were not conducted when the sea state was more than 5 (Beaufort scale 0 to 12) and during foggy days (visibility $<300 \mathrm{~m}$ ). Morning and evening glare were avoided by shifting to the side of the ship (starboard or port) opposite to the Sun.

\section{Aerial Surveys}

Aerial surveys were conducted at Larsemann hills and India Bay (Princess Astrid Coast) region to estimate the distribution and population of ice-breeding seals and penguins in the Indian sector of operation in 


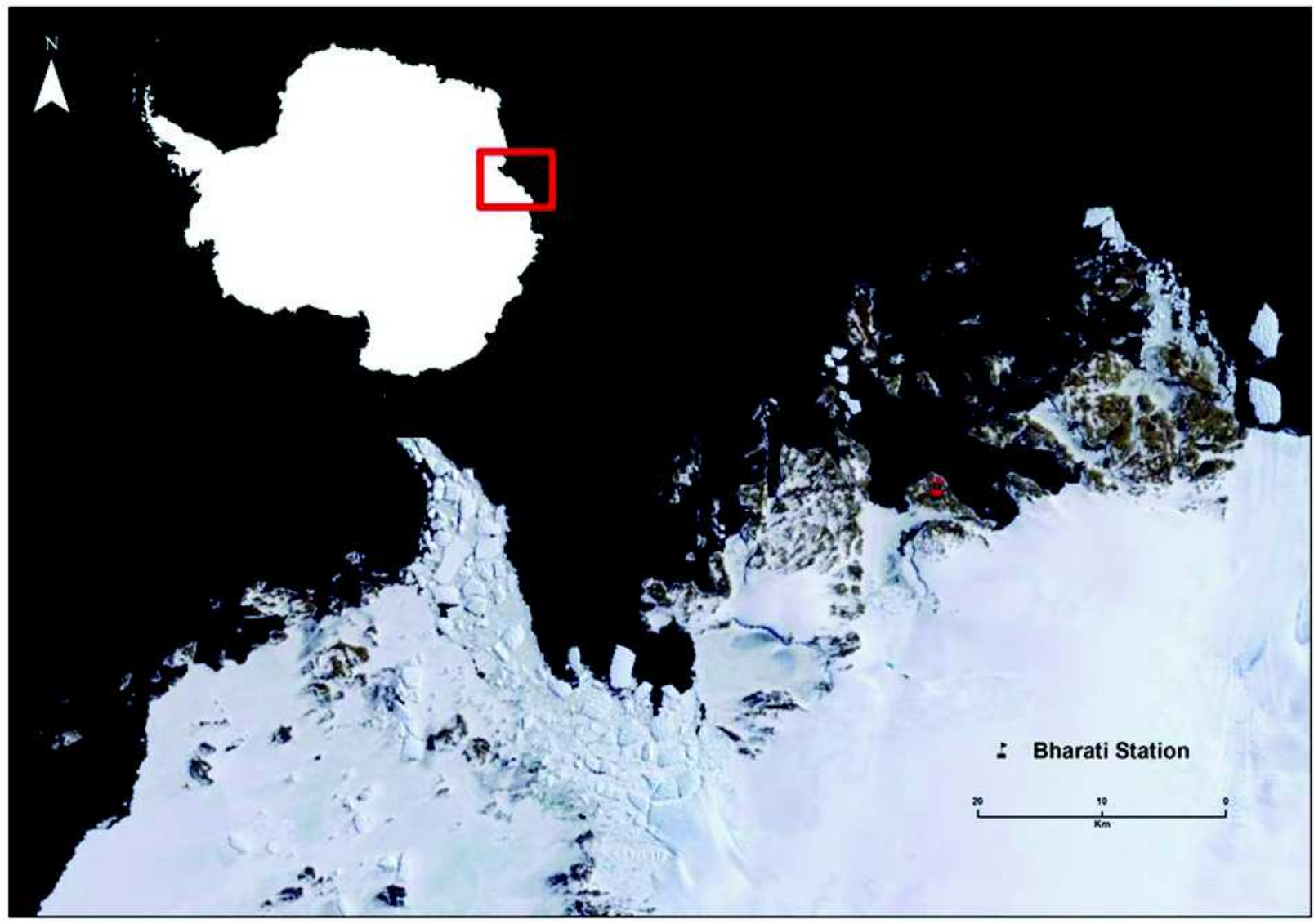

Fig. 2: Larsemann hills, east Antarctica, and site of Indian research station Bharati

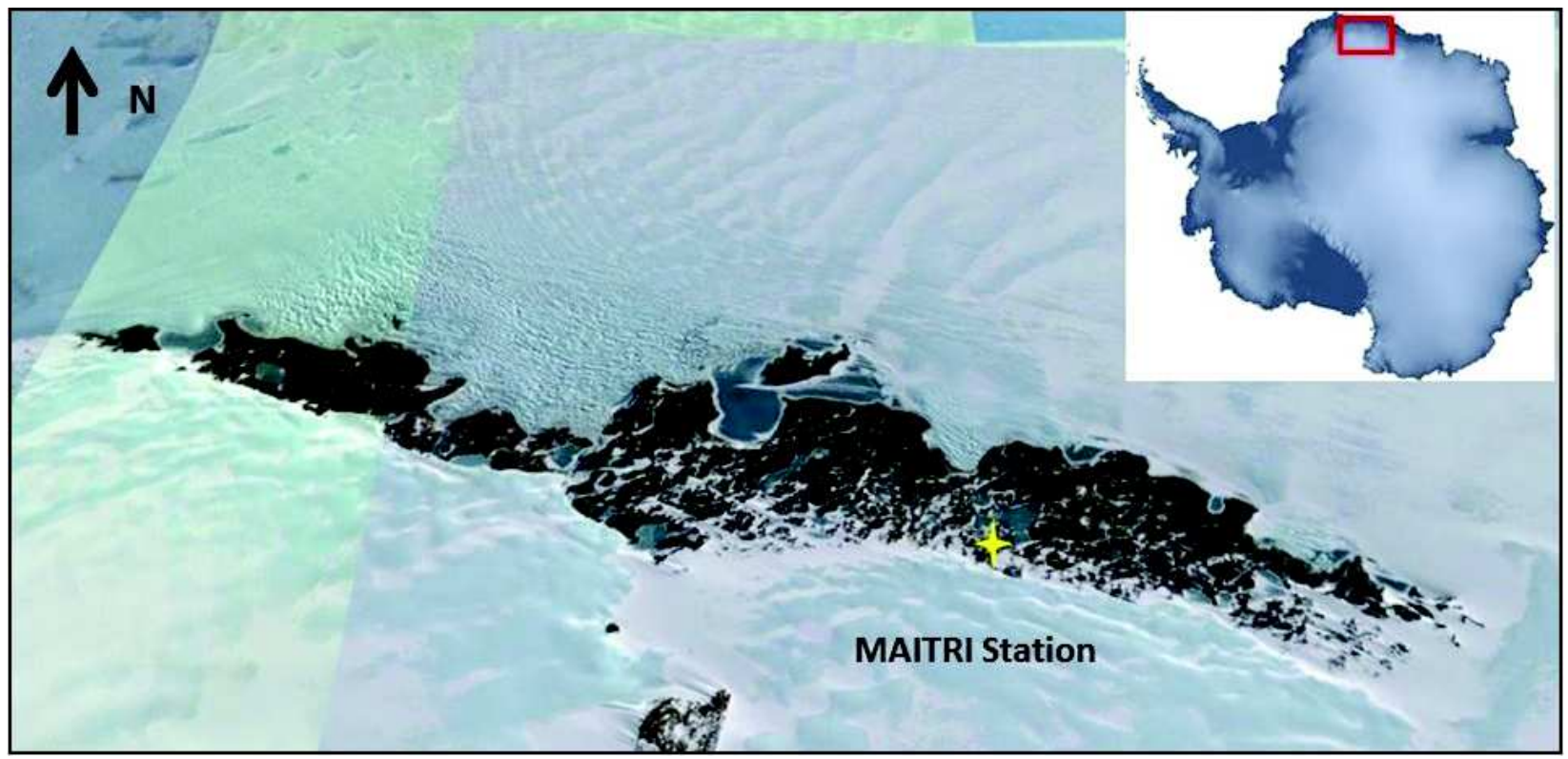

Fig. 3: Schirmacher oasis, central Dronning Maud Land, site of Indian research station Maitri

Antarctica. Aerial sorties were made on the Bell or Squirrel type helicopters in four expeditions' viz. $28^{\text {th }}$, 29 th, $33^{\text {rd }}$ and $35^{\text {th }}$ InSEAs. The methodology varied between expeditions due to modification of objectives in the last two expeditions. In the first two expeditions, surveys followed a pattern of flying along the contour 


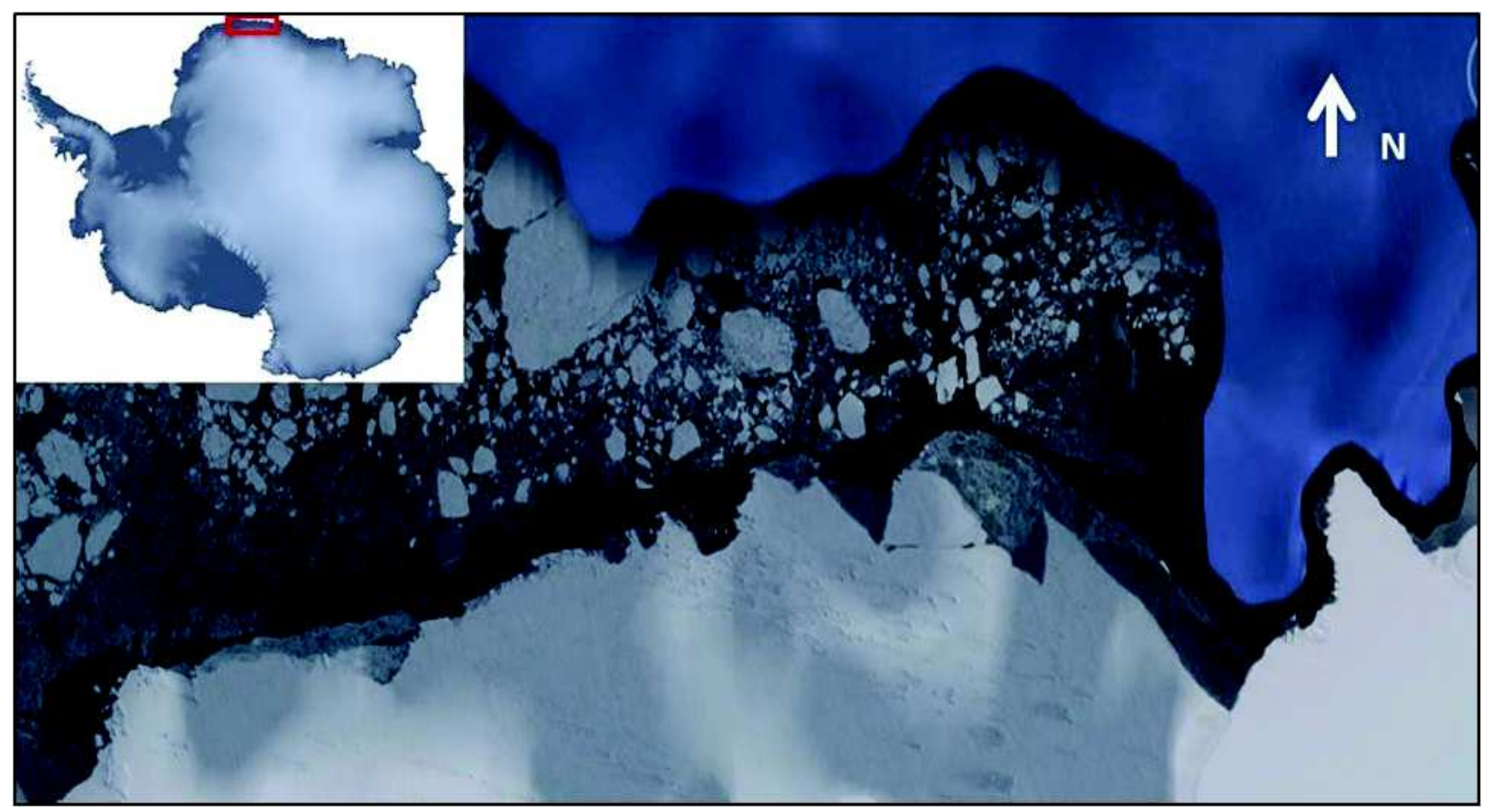

Fig. 4: India Bay, Princess Astrid coast

of the coastline, pack ice and fast ice (Sivakumar and Sathyakumar 2012; Kumar and Johnson 2014). In the last two, transects were laid, perpendicular from the coastline up to the edge of fast ice. A slight modification was made in the $35^{\text {th }}$ InSEA where high resolution video-documentation was employed to record ice-breeding seals in Larsemann hills region. All identified major coastal habitats, i.e. pack ice, fast ice, ice-free islands and near ice shelf zones were surveyed. In both methods, two observers scanned up to a visually estimated distance of $300 \mathrm{~m}$ on either side of the helicopter for hauled-out seals. The helicopter was flown at a uniform ground speed of 80 $\mathrm{km} \mathrm{h}^{-1}$ at an altitude of around $100 \mathrm{~m}$ above sea surface. Information on species, number, age class (adult or pup/chick), time, approx. distance from the transect line, reaction to the noise of helicopter, GPS location, etc. were subsequently recorded on each sighting. The sightings were also supplemented by aerial photography with Nikon D300 DSLR camera with Nikkor 70-300 mm zoom lens.

\section{On-foot Surveys}

Several islands at Larsemann hills were surveyed on foot to determine presence of any animal use. The smaller islands $(<2 \mathrm{sq} . \mathrm{km})$ were surveyed completely while straight line transects were conducted on larger islands to maximize efforts. This intensive area search method was also employed in, Schirmacher oasis which was surveyed on foot for detecting any animal presence. GPS locations were taken of seabird nest sites, feeding sites (in case of South Polar Skua), Adelie penguin moulting sites, direct sighting, dead remains and related information on species, habitat etc. were duly collected.

\section{Nest Monitoring}

Seabird species such as snow petrel Pagodroma nivea, south polar Skua Stercorarius maccormicki and Wilson's storm petrel Oceanites oceanicus were selected for long-term nest monitoring. The monitoring planned to cover all phases of nesting of the species which starts from November (egg-laying) and ends in February-March (fledging).

Snow petrel colonies were identified from onfoot surveys conducted at the islands based on presence of nests cavities with egg/chick/broken egg shells. Selected nesting sites were chosen for intensive monitoring of nesting behavior on the basis of their accessibility.

Study plots, each of $3 \times 3 \mathrm{~m}$, enough to cover many cavities which average about $0.2 \mathrm{~m}^{2}$ in entrance area (Einoder et al., 2014), were placed at fixed 
intervals along lines running diagonally from the bottom to the top of the colony (Mehlum et al., 1988). Each potential nest cavity within the study plot was marked using non-toxic paint and its geographic co-ordinates were recorded on a handheld GPS unit.

Intensive study plots $(3 \times 3 \mathrm{~m})$ were chosen from differently sized colonies to study nest cavity characteristics. The cavities were classified as occupied and unoccupied on the basis of presence of the bird. Physical characteristics such as nest depth, entrance height, entrance width and nest chamber volume were manually measured.

\section{Results}

\section{Seabird and Marine Mammal Observation Surveys}

A total of 15 vessel-based surveys resulted in $~ 384$ hours of observation in the aforementioned sectors during the Indian expeditions (Table 1) conducted over periods of Jan-Mar. 2009, Dec 2009-Mar. 2010, Dec. 2013-Apr 2014, Jan. 2015-Mar. 2015 and Feb. 2016. Forty-nine species of pelagic seabirds were recorded during the study period spread across the five

Table 1: Voyage survey details for the Indian Scientific Expeditions to Antarctica

\begin{tabular}{lcccc}
\hline Expedition & $\begin{array}{c}\text { Voyage } \\
\text { ID }\end{array}$ & $\begin{array}{c}\text { Time } \\
\text { period }\end{array}$ & $\begin{array}{c}\text { Sector } \\
\text { covered }\end{array}$ & $\begin{array}{c}\text { Effort } \\
\text { (in hours) }\end{array}$ \\
\hline 28th InSEA & V1 & Jan 2009 & CT-LH & 65.5 \\
& V2 & Feb 2009 & LH-IB & 36.0 \\
& V3 & Mar 2009 & IB- CT & 36.2 \\
29th InSEA & V4 & Dec 2009 & CT-LH & 44.0 \\
& V5 & Feb 2010 & LH-IB & 16.0 \\
& V6 & Mar 2010 & IB-CT & 40.6 \\
33rd InSEA & V7 & Dec 2013 & CT-LH & 19.5 \\
& V8 & Jan 2014 & LH-IB & 15.7 \\
& V9 & Feb 2014 & IB- CT & 24.7 \\
& V10 & Feb 2014 & CT-LH & 10.7 \\
& V11 & Mar 2014 & LH-IB & 12.0 \\
& V12 & Apr 2014 & IB- CT & 26.3 \\
& V13 & Jan 2015 & CT-LH & 25.5 \\
& V14 & Mar 2015 & IB- CT & 2.2 \\
34th InSEA & LH-IB & 8.7 \\
\hline 35th InSEA & V15 & Feb 2016 & LH
\end{tabular}

CT = Cape Town, South Africa, LH = Larsemann Hills, Antarctica, IB = Indian barrier (India Bay), Princess Astrid Coast expeditions (Table 2). Amongst the recorded species, four species such as Atlantic petrel, Grey-headed Albatross, Sooty Albatross and Indian Yellow-nosed Albatross are enlisted as endangered (IUCN 2016). Some seabird species were recorded only once, far from their actual distribution ranges, which could have been misidentified from morphologically similar species (Pande et al., 2014). Observations recorded were split into High Antarctic $\left(60^{\circ}\right.$ to $\left.70^{\circ} \mathrm{S}\right)$, Sub-Antarctic $\left(50^{\circ}\right.$ to $\left.60^{\circ} \mathrm{S}\right)$, Temperate $\left(40^{\circ}\right.$ to $\left.50^{\circ} \mathrm{S}\right)$ and Subtropical (north of $40^{\circ} \mathrm{S}$ ) oceanographic zones as per previous studies (Ribic et al., 2008; Commins et al., 2013). Encounter rates (birds seen $/ \mathrm{km}$ ) for the pelagic seabirds were found consistent for all the major oceanographic zones surveyed (Table 3 ). Bird densities peaked during the sub-Antarctic zone during early summers which shifted to high Antarctic zone during late summers (Fig. 5; Pande et al., 2015). The frequency of occurrence $(\%)$ of species in the subAntarctic and high Antarctic zone varied considerably within the austral summer season (Fig. 6).

Sixteen species of marine mammals were observed during vessel-based surveys in the southern Indian ocean. Out of these, about 11 species were seen exclusively in the High Antarctic zone (60-70 ${ }^{\circ} \mathrm{S}$ ) while two species (Humpback whale Megaptera novaeangliae and Sperm whale Physeter macrocephalus) were also seen north of $60{ }^{\circ} \mathrm{S}$ (see Table 4). Balaenopterids (baleen whales) formed the largest group (49\%) amongst all marine mammals dominated by Humpback whale (see Fig. 7).

\section{Aerial surveys at Larsemann hills and India Bay}

Aerial strip transects $(n=27$, effort $\sim 30$ hours $)$ resulted in a total of 1738 sightings (Table 5). 8822 individuals of four species of seals (Crabeater seal Lobodon carcinophaga, Leopard seal Hydrurga leptonyx, Ross seal Ommatophoca rossii \& Weddell seal Leptonychotesweddellii) and two penguin species (Adelie penguin Pygoscelis adeliae \& Emperor penguin Aptenodytes forsteri) were counted. Sightings were dominated by Weddell seal (58.2\% of overall sightings) at both India Bay and Larsemann hills survey areas (Fig. $8.1 \&$ 8.2). There was only one Ross seal sighting during the aerial surveys while pair of them was once seen hauled out on fast ice during voyage from Larsemann hills to India Bay (Kumar and Johnson, 2014). 
Table 2: Pelagic seabird species recorded during vessel-based surveys in the five InSEAs

\begin{tabular}{|c|c|c|c|c|c|c|c|c|c|c|}
\hline S.No & Common name & Scientific name & $\begin{array}{l}\text { IUCN } \\
\text { status }\end{array}$ & $\begin{array}{l}\mathrm{Pop}_{-} \\
\text {trend }\end{array}$ & $\begin{array}{c}2008- \\
09\end{array}$ & $\begin{array}{c}2009- \\
10\end{array}$ & $\begin{array}{c}2013- \\
14\end{array}$ & $\begin{array}{c}2014- \\
15\end{array}$ & $\begin{array}{c}2015- \\
16\end{array}$ & Zone \\
\hline 1 & Antarctic Fulmar & Fulmarus glacialoides & $\mathrm{LC}$ & stable & 1 & 1 & 1 & 1 & 1 & T,SA,HA \\
\hline 2 & Antarctic Petrel & Thalassoica antarctica & $\mathrm{LC}$ & stable & 1 & 1 & 1 & 1 & 1 & $\mathrm{~T}, \mathrm{SA}, \mathrm{HA}$ \\
\hline 3 & Antarctic Prion & Pachyptila desolata & $\mathrm{LC}$ & stable & 0 & 1 & 1 & 1 & 1 & T,SA,HA \\
\hline 4 & Antarctic tern & Sterna vittata & $\mathrm{LC}$ & unknown & 1 & 0 & 1 & 0 & 0 & $\mathrm{HA}$ \\
\hline 5 & Arctic tern & Sterna paradisaea & $\mathrm{LC}$ & decreasing & 0 & 1 & 0 & 1 & 0 & HA \\
\hline 6 & Atlantic Petrel & Pterodroma incerta & $\mathrm{EN}$ & decreasing & 1 & 0 & 1 & 0 & 0 & $\mathrm{~T}, \mathrm{SA}$ \\
\hline 7 & Black Petrel & Procellaria parkinsoni & VU & stable & 0 & 1 & 0 & 0 & 0 & ST,T,SA,HA \\
\hline 8 & Black-bellied Storm Petrel & Fregetta tropica & $\mathrm{LC}$ & decreasing & 1 & 0 & 1 & 1 & 0 & $\mathrm{~T}, \mathrm{SA}$ \\
\hline 9 & Black-browed Albatross & Thalassarche melanophris & $s \mathrm{NT}$ & decreasing & 1 & 1 & 1 & 0 & 0 & ST,T,SA \\
\hline 10 & Blue Petrel & Halobaena caerulea & $\mathrm{LC}$ & stable & 1 & 1 & 1 & 1 & 1 & $\mathrm{~T}, \mathrm{SA}, \mathrm{HA}$ \\
\hline 11 & Broad-billed Prion & Pachyptila vittata & $\mathrm{LC}$ & decreasing & 1 & 0 & 1 & 1 & 0 & ST,T,SA,HA \\
\hline 12 & Brown Skua & Catharacta antarctica & $\mathrm{LC}$ & stable & 1 & 0 & 1 & 0 & 0 & $\mathrm{~T}, \mathrm{HA}$ \\
\hline 13 & Cape Gannet & Morus capensis & VU & decreasing & 1 & 1 & 1 & 1 & 0 & $\mathrm{ST}, \mathrm{T}$ \\
\hline 14 & Cape Petrel & Daption capense & $\mathrm{LC}$ & stable & 1 & 1 & 1 & 1 & 1 & $\mathrm{~T}, \mathrm{SA}, \mathrm{HA}$ \\
\hline 15 & Common Diving Petrel & Pelecanoide surinatrix & $\mathrm{LC}$ & decreasing & 1 & 0 & 1 & 1 & 0 & $\mathrm{~T}, \mathrm{SA}, \mathrm{HA}$ \\
\hline 16 & Cory's Shearwater & Calonectris borealis & $\mathrm{LC}$ & unknown & 1 & 1 & 1 & 0 & 0 & ST,SA,SA \\
\hline 17 & Fairy Prion & Pachyptila turtur & $\mathrm{LC}$ & stable & 1 & 0 & 1 & 0 & 0 & $\mathrm{~T}, \mathrm{SA}, \mathrm{HA}$ \\
\hline 18 & Flesh-footed Shearwater & Ardenna carneipes & $\mathrm{LC}$ & stable & 1 & 0 & 1 & 0 & 0 & ST \\
\hline 19 & Great Shearwater & Ardenna gravis & $\mathrm{LC}$ & stable & 0 & 0 & 1 & 0 & 0 & ST \\
\hline 20 & Great-winged Petrel & Pterodroma macroptera & $\mathrm{LC}$ & decreasing & 1 & 1 & 1 & 1 & 0 & ST,T,SA,HA \\
\hline 21 & Grey Petrel & Procellaria cinerea & NT & decreasing & 1 & 1 & 1 & 0 & 0 & T,SA,HA \\
\hline 22 & Grey-backed Storm Petrel & Garrodia nereis & $\mathrm{LC}$ & decreasing & 1 & 0 & 0 & 0 & 0 & $\mathrm{~T}$ \\
\hline 23 & Grey-headed Albatross & Thalassarche chrysostoma & $a \mathrm{EN}$ & decreasing & 1 & 1 & 1 & 1 & 1 & ST,T,SA,HA \\
\hline 24 & $\begin{array}{l}\text { Indian Yellow-nosed } \\
\text { Albatross }\end{array}$ & Thalassarche carteri & $\mathrm{EN}$ & decreasing & 1 & 1 & 1 & 0 & 0 & ST,SA \\
\hline 25 & Kerguelen Petrel & Aphrodroma brevirostris & $\mathrm{LC}$ & stable & 1 & 0 & 1 & 1 & 0 & $\mathrm{~T}, \mathrm{SA}, \mathrm{HA}$ \\
\hline 26 & Laysan Albatross* & Phoebastria immutabilis & NT & stable & 1 & 0 & 0 & 0 & 0 & ST \\
\hline 27 & Leach's Storm Petrel* & Hydrobates leucorhous & $\mathrm{LC}$ & stable & 1 & 0 & 0 & 0 & 0 & ST \\
\hline 28 & Light-mantled Albatross & Phoebetria palpebrata & NT & decreasing & 1 & 1 & 1 & 1 & 1 & T,SA,HA \\
\hline 29 & Little Shearwater & Puffinus assimilis & LC & decreasing & 0 & 0 & 1 & 0 & 0 & $\mathrm{ST}, \mathrm{T}$ \\
\hline 30 & Northern Giant Petrel & Macronectes halli & $\mathrm{LC}$ & increasing & 0 & 1 & 1 & 1 & 1 & T,SA,HA \\
\hline 31 & Salvin's Albatross* & Thalassarche salvini & VU & unknown & 1 & 0 & 0 & 0 & 0 & ST \\
\hline 32 & Salvin's Prion & Pachyptila salvini & $\mathrm{LC}$ & stable & 0 & 1 & 1 & 0 & 0 & $\mathrm{~T}, \mathrm{SA}$ \\
\hline 33 & Short-tailed Shearwater & Ardennate nuirostris & $\mathrm{LC}$ & decreasing & 0 & 0 & 1 & 0 & 0 & ST,T,SA,HA \\
\hline 34 & Slender-billed Prion & Pachyptila belcheri & $\mathrm{LC}$ & stable & 1 & 0 & 1 & 1 & 0 & T,SA,HA \\
\hline 35 & Snow Petrel & Pagodroma nivea & $\mathrm{LC}$ & stable & 1 & 1 & 1 & 1 & 1 & HA \\
\hline 36 & Soft-plumaged Petrel & Pterodroma mollis & $\mathrm{LC}$ & stable & 1 & 1 & 1 & 1 & 0 & ST,T,SA \\
\hline 37 & Sooty Albatross & Phoebetriafusca & $\mathrm{EN}$ & decreasing & 1 & 1 & 1 & 1 & 0 & ST,T,SA,HA \\
\hline 38 & Sooty Shearwater & Ardenna grisea & $\mathrm{NT}$ & decreasing & 1 & 1 & 1 & 0 & 0 & ST,T,SA,HA \\
\hline 39 & South Polar Skua & Catharacta maccormicki & $\mathrm{LC}$ & stable & 1 & 1 & 1 & 1 & 1 & ST,T,SA,HA \\
\hline 40 & Southern Giant Petrel & Macronectes giganteus & $\mathrm{LC}$ & increasing & 1 & 1 & 1 & 1 & 1 & ST,T,SA,HA \\
\hline 41 & Southern Royal Albatross* & Diomedea epomophora & VU & stable & 1 & 0 & 1 & 1 & 0 & ST,T,SA,HA \\
\hline 42 & Wandering Albatross & Diomedea exulans & VU & decreasing & 1 & 1 & 1 & 1 & 0 & ST,T,SA,HA \\
\hline 43 & Wedge-tailed Shearwater* & Ardenna pacifica & $\mathrm{LC}$ & decreasing & 0 & 0 & 1 & 0 & 0 & SA \\
\hline 44 & Westland Petrel* & Procellaria westlandica & VU & stable & 0 & 0 & 1 & 0 & 0 & SA \\
\hline 45 & White-bellied Storm Petrel & Fregetta grallaria & $\mathrm{LC}$ & decreasing & 1 & 1 & 0 & 0 & 0 & ST,T,SA \\
\hline 46 & White-capped Albatross* & Thalassarche steadi & $\mathrm{NT}$ & decreasing & 1 & 0 & 0 & 0 & 0 & ST,T,SA \\
\hline 47 & White-chinned Petrel & Procellaria aequinoctialis & VU & decreasing & 1 & 1 & 1 & 1 & 1 & ST,T,SA,HA \\
\hline 48 & White-headed Petrel & Pterodroma lessonii & $\mathrm{LC}$ & decreasing & 1 & 1 & 1 & 1 & 0 & ST,T,SA,HA \\
\hline 49 & Wilson's Storm Petrel & Oceanites oceanicus & $\mathrm{LC}$ & stable & 1 & 1 & 1 & 1 & 1 & ST,T,SA,HA \\
\hline
\end{tabular}

1 - Recorded, 0 - Not recorded, ST = Sub-tropical, T - Temperate, SA - Sub-Antarctic, HA - High Antarctic, EN - Endangered, LC Least Concern, NT - Near Threatened, VU - Vulnerable (Threat categories of IUCN Red List), *Probably misidentified or vagrant

\#Source: IUCN Red List accessed on 15th July 2016 
Table 3: Encounter rates (birds/km) for the seabirds recorded in major oceanographic zones in the southern Indian Ocean in the past five expeditions

\begin{tabular}{lcccc}
\hline InSEA & High antarctic & Sub-antarctic & Temperate & Sub-tropical \\
\hline 28 & $0.16 \pm 0.52$ & $0.15 \pm 0.34$ & $0.07 \pm 0.1$ & $0.09 \pm 0.23$ \\
29 & $0.30 \pm 0.61$ & $0.36 \pm 0.78$ & $0.11 \pm 0.2$ & $0.04 \pm 0.03$ \\
33 & $1.30 \pm 3.32$ & $1.07 \pm 2.74$ & $1.51 \pm 10.61$ & $0.63 \pm 1.13$ \\
34 & $1.13 \pm 2.42$ & $2.99 \pm 7.93$ & $0.28 \pm 0.17$ & $0.32 \pm 0.18$ \\
35 & $0.69 \pm 1.46$ & $\mathrm{ND}$ & $\mathrm{ND}$ & $\mathrm{ND}$ \\
\hline
\end{tabular}

Table 4: Marine mammal species seen during vessel-based surveys in the southern Indian ocean. Percentage abundance of each species is for the particular zone where it was sighted. This does not include the unidentified sightings

\begin{tabular}{|c|c|c|}
\hline Zone & Species & $\%$ Abundance \\
\hline \multirow[t]{2}{*}{ Sub-tropical } & Sperm Whale & 0.84 \\
\hline & Long-finned Pilot Whale & 35.49 \\
\hline \multirow[t]{3}{*}{ Temperate } & Dusky Dolphin & 0.84 \\
\hline & Sperm Whale & 0.21 \\
\hline & Long-finned Pilot Whale & 1.67 \\
\hline \multirow[t]{2}{*}{ Sub-Antarctic } & Antarctic fur seal & 0.21 \\
\hline & Humpback Whale & 0.63 \\
\hline \multirow[t]{13}{*}{ High Antarctic } & Blue whale & 0.21 \\
\hline & Leopard Seal & 0.21 \\
\hline & Ross seal & 0.21 \\
\hline & Southern bottlenose whale & 0.21 \\
\hline & Minke Whale & 1.67 \\
\hline & Sei Whale & 1.67 \\
\hline & Weddell Seal & 2.09 \\
\hline & Sperm Whale & 2.30 \\
\hline & Killer whale & 3.76 \\
\hline & Antarctic Minke Whale & 4.59 \\
\hline & Fin whale & 5.64 \\
\hline & Crabeater Seal & 10.65 \\
\hline & Humpback Whale & 26.93 \\
\hline
\end{tabular}

\section{Distribution of Wildlife Around Indian Stations}

Bharati station, Larsemann Hills: Foot surveys carried out in the Larsemann Hills region revealed presence of five bird species, viz. Adelie penguin, emperor penguin, snow petrel, south polar Skua and Wilson's storm petrel (Pande et al., 2014). In all, 15 island/peninsula were surveyed for the presence of habitat use by the birds, of which, nesting sites were detected in 14 islands (Table 6).

Maitri Station, Schirmacher Oasis: Six breeding pairs of south polar Skua were observed in the eastern part of the oasis. Dead remains of two species, Adelie penguin and snow petrel were also found in this area. There was also a single sighting of the Wilson's storm petrel in the area north of the station. The total Skua population on the eastern side of Maitri could be somewhere around 12-15 individuals. Most of these individuals were habituated to human presence and were found to roost near the Indian and Russian stations in the oasis. Four south polar Skua individuals were also ringed with colored leg bands for long-term monitoring of breeding pairs near Maitri station.

\section{Preliminary Results From Nest Monitoring}

Over 200 nests of snow petrel were marked and measured for long-term monitoring at Larsemann hills during the last two austral summers (2014-15 \& 201516). Initial analysis from the cavity parameters classified nest cavities of snow petrels in three types viz. boulder, crack and slab (Pande et al., 2015). Out of these three, slab type cavity was the preferred site for nesting accounting for close to $60 \%$ of the nests. The cavities with breeding pairs were also lower in volume and narrower compared to the unoccupied cavities (Fig. 9 A \& B).

Table 5: Aerial surveys conducted during the Indian Scientific Expeditions to Antarctica*

\begin{tabular}{lcllc}
\hline Expedition & Aerial surveys (n) & Time period & Sector covered & Effort (in hours) \\
\hline $28^{\text {th }}$ InSEA & 7 & Jan-Feb 2009 & Larsemann Hills \&Princess Astrid coast & 6.69 \\
$29^{\text {th }}$ InSEA & 6 & Dec 2009-Feb 2010 & Larsemann Hills \&Princess Astrid coast & 10.67 \\
$33^{\text {rd }}$ InSEA & 11 & Dec 2013-Mar 2014 & Larsemann Hills \&Princess Astrid coast & 9.55 \\
$35^{\text {th }}$ InSEA & 3 & Jan-Feb 2016 & Larsemann Hills & 2.99 \\
\hline
\end{tabular}

\footnotetext{
*No aerial surveys were conducted in $34^{\text {th }}$ InSEA (2014-15) due to unavailability of helicopter support
} 
Table 6: Status of seabirds in islands of Larsemann hills

\begin{tabular}{llcccc}
\hline S.No. & Island/Peninsula & Adelie Penguin & Snow Petrel & South Polar Skua & Wilson's Storm Petrel \\
\hline 1 & Betts & $\mathrm{M}$ & $\mathrm{N}$ & $\mathrm{N}$ & + \\
2 & Grovnes & $\mathrm{M}$ & $\mathrm{N}$ & $\mathrm{N}$ & $\mathrm{N}$ \\
3 & Breadloaf & - & $\mathrm{N}$ & + & - \\
4 & Broknes & - & $\mathrm{N}$ & + & $\mathrm{N}$ \\
5 & Butler & $\mathrm{M}$ & & $\mathrm{N}$ & $\mathrm{N}$ \\
6 & Easther & $\mathrm{M}$ & $\mathrm{N}$ & + & $\mathrm{N}$ \\
7 & Fisher & $\mathrm{M}$ & $\mathrm{N}$ & + & $\mathrm{N}$ \\
8 & Harley & $\mathrm{M}$ & - & - & - \\
9 & John & - & - & $\mathrm{N}$ & - \\
10 & Manning & $\mathrm{M}$ & $\mathrm{N}$ & $\mathrm{N}$ & $\mathrm{N}$ \\
11 & Solomon & $\mathrm{M}$ & - & - & $\mathrm{N}$ \\
12 & Cook & $\mathrm{M}$ & $\mathrm{N}$ & - & $\mathrm{N}$ \\
13 & Osmar & $\mathrm{M}$ & - & + & - \\
14 & Sandercock & - & - & $\mathrm{N}$ & - \\
15 & McLeod & $\mathrm{M}$ & $\mathrm{N}$ & $\mathrm{N}$ & $\mathrm{N}$ \\
\hline
\end{tabular}

$+=$ present but no nesting, - = Not detected, $\mathrm{M}=$ Moulting, $\mathrm{N}=$ Nesting (updated from Pande et al., 2014)

\section{Discussion}

\section{Population Assessments}

This study generated baseline data on key seabird and marine mammal species in the southern Indian ocean and especially in Prydz bay and India bay. The seabird counts from the vessel-based surveys indicate higher bird species richness in the sub-Antarctic zone gradually declining towards the high Antarctic. However, the bird abundance displayed an increasing trend as expected towards the continental waters (Fig. 5). The increase in bird abundance in the high Antarctic during late summers is attributed to the retreating sea ice towards the coastal shelf areas (Commins et al., 2013). Birds which are abundant in the high Antarctic zone had a relatively higher frequency of occurrence during late summer observations (Fig. 6). Birds such as Arctic tern Sterna paradisaea, blue petrel Halobaena caerulea, snow petrel Pagodroma nivea and light-mantled albatross Phoebetria palpebrata moved towards open waters south of $60^{\circ} \mathrm{S}$ in the late summers. Arctic terns are known to forage in the high Antarctic zone from December to March (Egevang et al., 2010) whereas surface-seize feeders such as blue petrel, snow petrel and light-mantled albatross prefer foraging in open water polynyas within the sea ice zone (Ainley et al.,
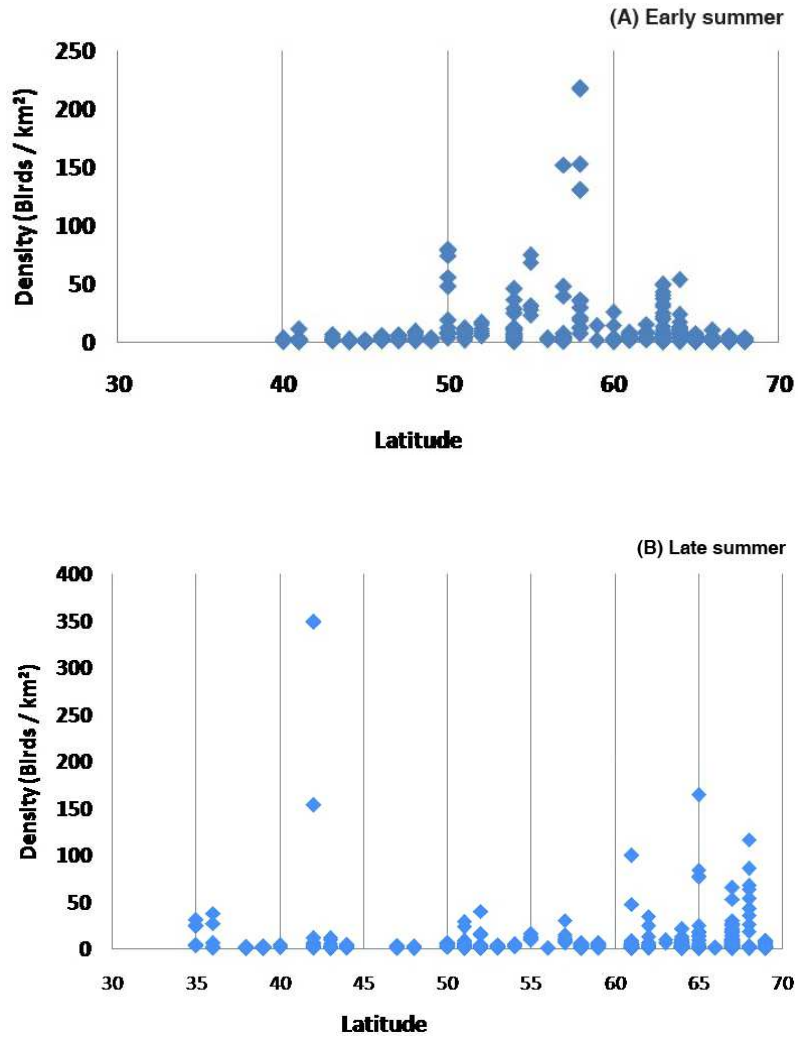

Fig. 5: Density of pelagic seabirds during the austral summers. (A) early summer, (B) late summer (Pande et al., 2015) 


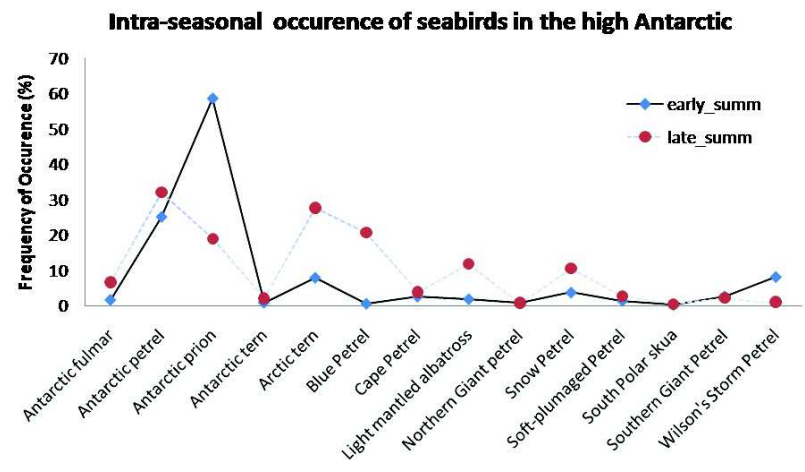

Fig. 6: Intra-seasonal occurrences of key pelagic species in the high Antarctic (60-70 $\mathrm{S})$

2003; Ribic et al., 2008). In the high Antarctic zone, mixed feeders increased in proportion as compared to the plankton, fish and cephalopod feeders (Pande et al., 2014). This change is duly attributed to Antarctic petrel and Antarctic prion which were seen in large flocks during the voyage surveys. Similarly, baleen whale species such as Humpback whales spend considerable amount of time foraging in the nutrientrich waters of the southern ocean during the austral summer (Robbins et al., 2011; Constantine et al., 2014) to feed on abundant Antarctic krill swarms (Siegel et al., 2013). Species-specific distribution maps would be helpful in understanding seabird movements in relation to retreating sea ice. A comprehensive monitoring protocol for the seabirds during In SEAs will be prepared after complete analysis of data gaps.

Weddell seal was found to be the dominant species of the fast ice areas of the Indian sector of operation in Antarctica. The estimates derived from previous counts (Sathyakumar 1995; Bhatnagar and Sathyakumar 1997; Hussain and Saxena 2008) were higher in comparison to past five years data (Table 7). This is probably due to a change in methodology and the area covered by the aerial surveys. Counts of Weddell seals in Larsemann hills were higher than that on Princess Astrid coast due to persistent fast ice in embayment areas in the Prydz bay which accounted for more number of seals hauled out during the surveys (Sivakumar and Sathyakumar 2012; Kumar and Johnson 2014).

\section{Habitat Assessments}

Surveys to determine habitat use by seabirds in
Larsemann hills covered all major islands and peninsulas except Stornes. Stornes peninsula $\left(69^{\circ} 25^{\prime} \mathrm{S}, 7^{\circ} 6^{\prime} \mathrm{E}\right)$ being an Antarctic Specially Protected Area no. 174 (ATS 2014) was not sampled for seabird distribution. Islands/peninsulas like McLeod, Manning and Broknes which are comparatively larger in size could not be covered entirely due to time constraint and inaccessibility. South polar Skua nesting and feeding sites were found in close proximity to snow petrel colonies in islands such as Easther. However, only spatial mapping would reveal any significant relationship between habitat useof these two species. In Schirmacher oasis, the nesting of south polar Skuas was observed to be positively influenced by human presence. Further monitoring of skua breeding pairs is needed using radiotelemetry to understand their movement patterns and territoriality during the breeding period. Dead remains of Adelie penguin suggest the movement of penguins from one colony to another through the oasis which can be confirmed using molecular analysis or radiotelemetry of passing individuals.

\section{Conclusion}

Several studies in the past have highlighted the role played by seabirds and marine mammals in maintaining the Antarctic ecosystem health (Croxall et al., 2002; Weimerskirch et al., 2003; McMahon \& Burton 2005). The CCAMLR Ecosystem Monitoring Program (CEMP) also emphasizes the importance of monitoring key seabird and marine mammal species to serve as a basis for the conservation of Antarctic marine living resources and understand the physical and biological drivers of population changes. With data available from several years of monitoring identified species and habitats, we would be better able to understand sensitive species and populations to environmental fluctuations and changes in ecological parameters (such as breeding phenology) over time. Baseline data generated from this study would form the bedrock of the future detailed investigations on demographic assessments, genetic studies and ecosystem monitoring.

In summary, data on distribution patterns of oceanic birds, seals and penguins in the Indian sector of operation would help in preparing monitoring protocols in view of changing climate. The nest monitoring study backed by past survey data will help 
Table 7: Abundance estimates of some key species based on wildlife monitoring studies done by Wildlife Institute of India in Indian area of operation in Antarctica

\begin{tabular}{|c|c|c|c|c|c|c|c|c|c|c|c|c|}
\hline & \multicolumn{3}{|c|}{ Weddell Seal } & \multicolumn{3}{|c|}{ Crabeater Seal } & \multicolumn{3}{|c|}{ Adelie Penguin } & \multicolumn{3}{|c|}{ Emperor Penguin } \\
\hline & $\mathrm{SC}$ & ER & GS & $\mathrm{SC}$ & ER & GS & $\mathrm{SC}$ & ER & GS & $\mathrm{SC}$ & ER & GS \\
\hline *Sathyakumar 1995 & - & - & - & 17.8 & $1.04 \pm 0.51$ & - & 34.8 & $1.80 \pm 1.62$ & - & 47.2 & $3.16 \pm 2.75$ & - \\
\hline *Bhatnagar \& Sathyakumar 1997 & - & $1.1 \pm 1.1$ & - & - & $1.1 \pm 0.6$ & - & - & $1.9 \pm 1.0$ & - & - & $3.5 \pm 2.3$ & - \\
\hline *Hussain \& Saxena 2008* & 90.2 & $6.57 \pm 1.27$ & $6.0 \pm 1.2$ & 8.3 & - & $1.82 \pm 0.1$ & 30.0 & $12.28 \pm 4.97$ & $10.6 \pm 7.8$ & 70.0 & $29.5 \pm 7.3$ & $34.0 \pm 20.4$ \\
\hline \#Sivakumar \& Sathyakumar 2012 & $\begin{array}{l}\text { a9 } 90.0^{\wedge} \\
\text { b5 } 53.0^{\wedge}\end{array}$ & $1.99 \pm 1.41^{\wedge}$ & $6.32 \pm 7.75$ & b8. $80^{\wedge}$ & $0.10 \pm 0.10^{\wedge}$ & $1.69 \pm 1.20$ & $\begin{array}{l}\text { a } 6.0^{\wedge} \\
\text { b9. } 9.0^{\wedge}\end{array}$ & $\begin{array}{l}\text { a24.0 } 21.0^{\mathrm{b}} \\
23.0 \pm 19.0\end{array}$ & $13.0 \pm 10.0$ & $\begin{array}{l}5.35 \pm 8.43 \\
5.32 \pm 7.89\end{array}$ & $\begin{array}{l}{ }^{\mathrm{a}} 1.0^{\wedge} \\
\mathrm{b} 17.0^{\wedge}\end{array}$ & ${ }^{\mathrm{a}} 2.0 \pm 1.0^{\mathrm{b}}$ \\
\hline \#Kumar \& Johnson 2014 & 97.0 & $4.02 \pm 2.95$ & $4.71 \pm 5.50^{\wedge}$ & 1.7 & $\begin{array}{l}0.001 \pm \\
0.002\end{array}$ & $1.46 \pm 0.76^{\wedge}$ & 9.77 & $0.44 \pm 0.33^{\wedge}$ & $4.96 \pm 5.51^{\wedge}$ & 23.15 & $1.05 \pm 0.84^{\wedge}$ & $0.0 \pm 21.3^{\wedge}$ \\
\hline \#Pande et al. 2014 & 42.2 & $0.47 \pm 0.48^{\wedge}$ & $2.58 \pm 2.64$ & 18.9 & $0.15 \pm 0.16^{\wedge}$ & $2.96 \pm 3.14$ & 26.8 & $0.92 \pm 2.1$ & $2.77 \pm 3.28^{\wedge}$ & 1.8 & $0.04 \pm 0.03^{\wedge}$ & $2.14 \pm 1.86$ \\
\hline \$This study (2008-2016) & 54.0 & $8.52 \pm 8.20$ & $4.68 \pm 5.81$ & 2.0 & $0.51 \pm 0.53$ & $1.68 \pm 1.66$ & 11.0 & $2.31 \pm 1.75$ & $4.25 \pm 5.16$ & 17.0 & $2.50 \pm 2.63$ & $8.61 \pm 17.60$ \\
\hline
\end{tabular}

SC - Species composition (\% abundance), ER - Encounter rates (individuals seen $/ \mathrm{km}) \pm \mathrm{SD}, \mathrm{GS}$ - Group Size \pm SD

^unpublished data, ${ }^{\mathrm{a} L a r s e m a n n}$ hills, ${ }^{\mathrm{b}}$ India Bay,

*Studies conducted in India bay, "Studies conducted in both Larsemann hills and India bay sectors

${ }^{\$}$ Combined estimates of studies conducted in the past four expeditions i.e. $28^{\text {th }}, 29^{\text {th }}, 33^{\text {rd }} \& 35^{\text {th }}$
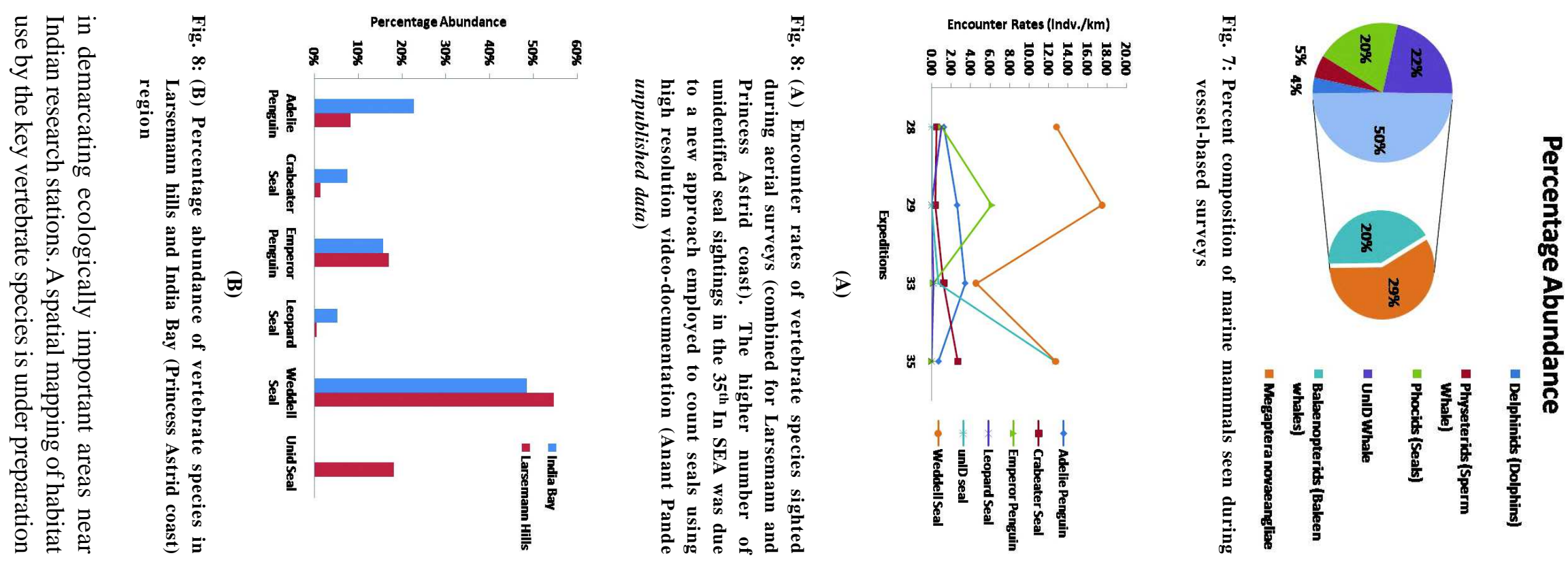


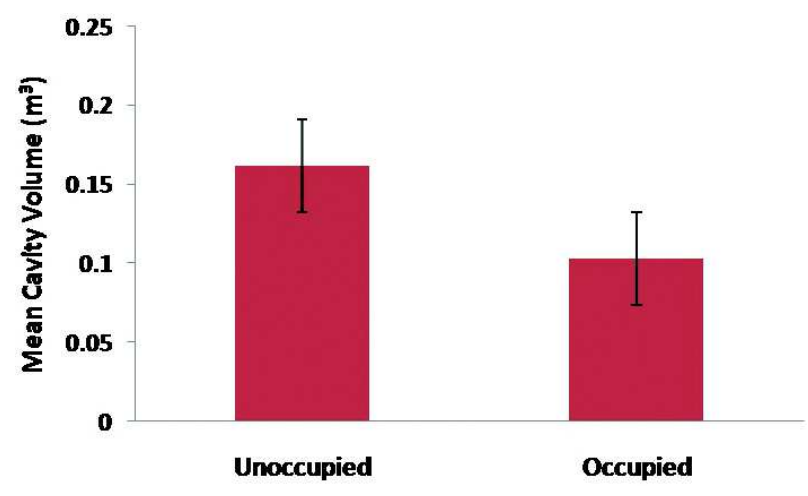

(A)

Fig. 9: (A) Nest cavity volume of snow petrel nests marked in Larsemann hills for long-term monitoring

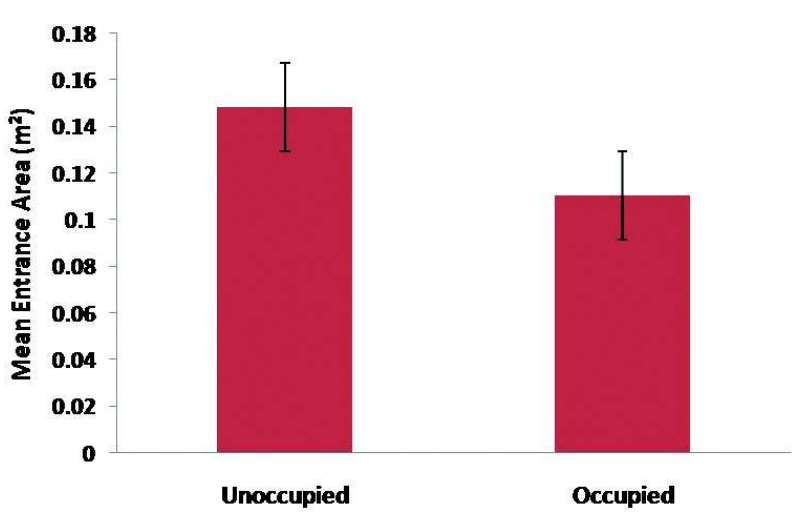

(B)

Fig. 9: (B) Nest entrance area of snow petrel nests marked in Larsemann hills for long-term monitoring
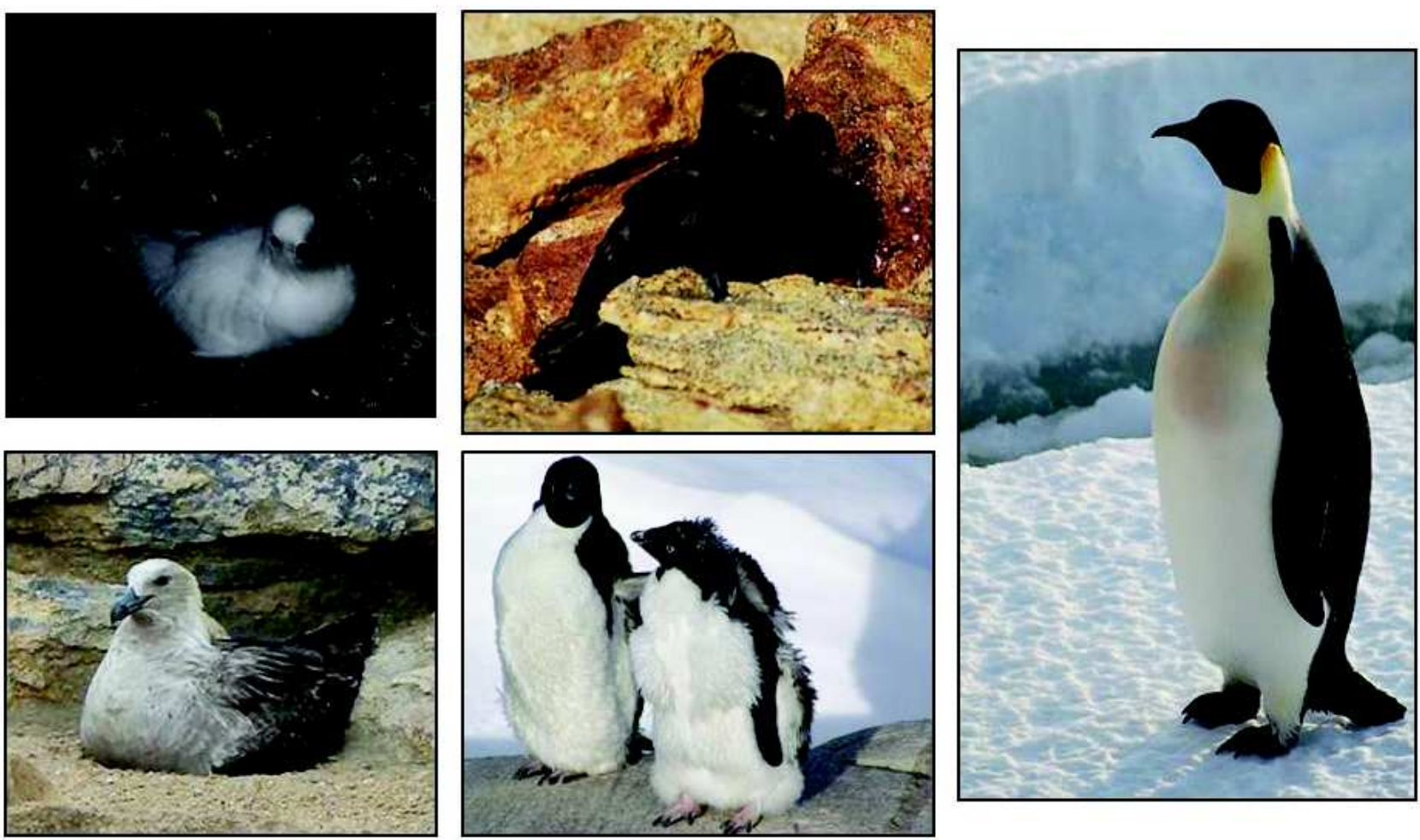

Plate 1: Seabird species occurring in Larsemann hills, Prydz bay, Antarctica. Clockwise from top left: Snow petrel, Wilson's Storm petrel, Emperor penguin, Adelie penguin, South polar Skua

to inform station activity planning and establish monitoring protocols for further studies on behaviour, genetic structuring and population monitoring.

\section{Acknowledgements}

We are grateful to the ESSO-National Centre for Antarctic and Ocean Research, Goa for providing logistic support during the Indian Scientific Expeditions to Antarctica. Sincere thanks to Mirza Javed Beg, Rahul Mohan (ESSO -NCAOR) and Dean, WII for their constant support and encouragement. We are also thankful to the team members of Indian Scientific Expedition to Antarctica for their support and especially Subrata Gouda, Vineeth Vasudevan (NHO) for their help during the data collection. 

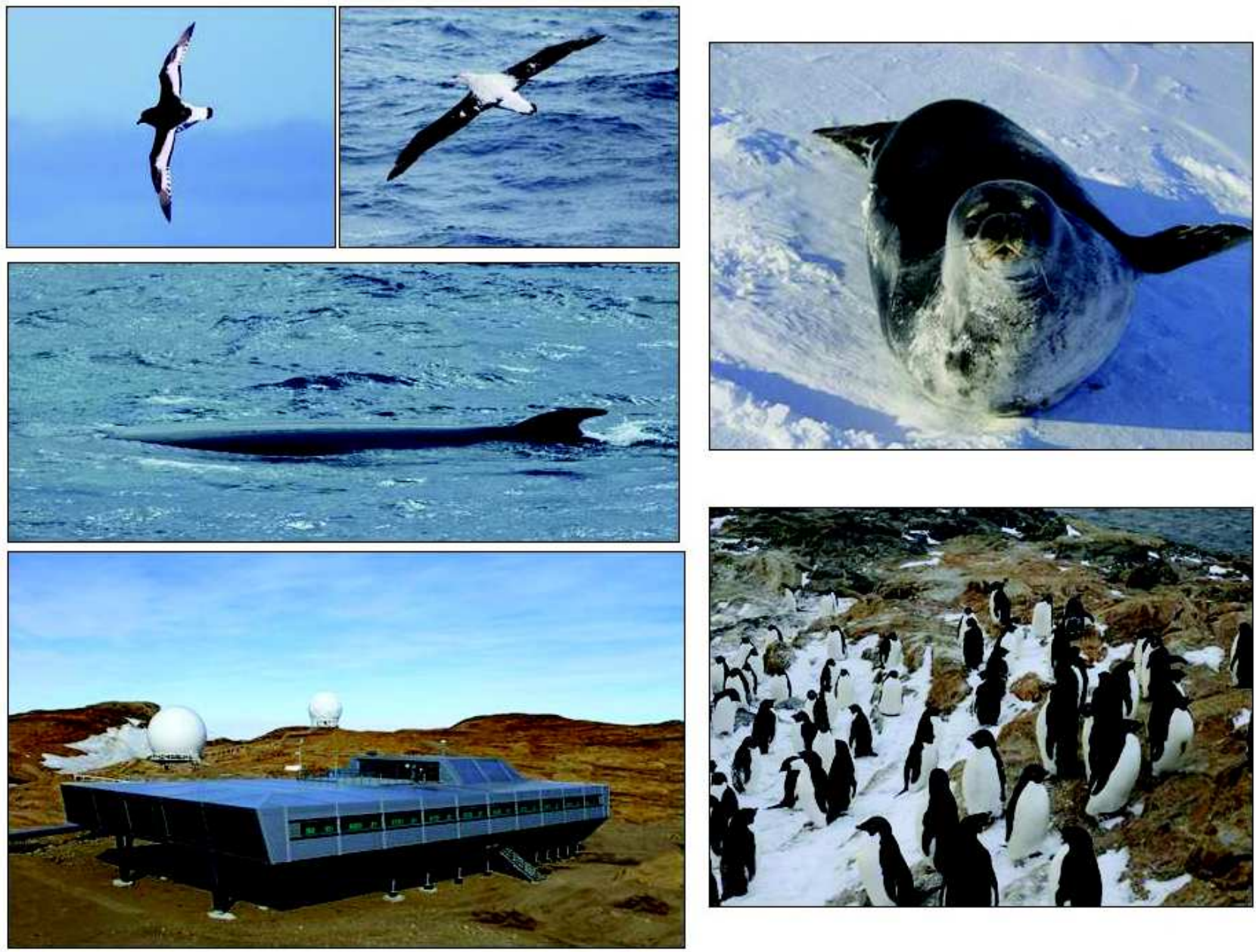

Plate 2: Some commonly seen species during Indian Antarctic expeditions. Clockwise from top left: Antarctic petrel, Wandering Albatross, Weddell seal, Adelie penguin aggregation; Bharati research station at Larsemann hills, Antarctica; and Antarctic Minke whale

\section{References}

Abrams R W (1985) Environmental determinants of pelagic seabird distribution in the African sector of the Southern Ocean $J$ Biogeo 473-492

Ainley D G, Tynan C T and Stirling I (2003) Sea ice: a critical habitat for polar marine mammals and birds. In Thomas DN, Diekman GS (Eds) Sea Ice: An Introduction to its Physics, Biology, Chemistry and Geology. Blackwell Science, London, pp 240-266

ATS (2016) - Measure 2 (2007) Annex B Management Plan for Antarctic Specially Managed Area No. 6 Larsemann hills, east Antarctica http://www.ats.aq/documents/recatt/ Att358_e.pdf- Accessed on 20 July 2016

Agustí, Sejr M K and Duarte C M (2010) Impacts of climate warming on polar marine and freshwater ecosystems Polar Biol 33 1595-1598
Bhatnagar Y V and Sathyakumar S (1999) Daily monitoring and aerial census of penguins and seals in Antarctica.Technical Publication Department of Ocean Development 13 165182

Bost C A, Cotté C, Bailleul F et al. (2009) The importance of oceanographic fronts to marine birds and mammals of the southern oceans J Marine Syst 78 363-376

Coetzee B W and Chown S L (2015) A meta analysis of human disturbance impacts on Antarctic wildlife Biol Rev 91 578596

Commins M L, Ansorge I and Ryan P G (2013) Multi-scale factors influencing seabird assemblages in the African sector of the Southern Ocean Antarct Sci 26 38-48

Constable A J, Melbourne Thomas J, Corney S P et al. (2014) Climate change and Southern Ocean ecosystems I: how changes in physical habitats directly affect marine biota 


\section{Glob Chang Biol 20 3004-3025}

Constantine R, Steel D, Allen J et al. (2014) Remote Antarctic feeding ground important for east Australian humpback whales Marine Bioly 161 1087-1093

Croxall J P, Trathan P N and Murphy E J (2002) Environmental change and Antarctic seabird populations Science 297 15101514

Egevang C, Stenhouse I J, Phillips R A et al. (2010) Tracking of Arctic terns Sterna paradisaea reveals longest animal migration PNAS 107 2078-2081

Einoder L D, Emmerson L M, Southwell D M and Southwell C J (2014) Cavity characteristics and ice accumulation affect nest selection and breeding in snow petrels (Pagodro manivea), Marine Ornith 42 175-182

Hansen J E, Ruedy R, Glascoe J and Sato M (1999) GISS analysis of surface temperature change J Geophys Res 10430997 31022

Hodgson D A, Verleyen E, Vyverman W et al. (2009) A geological constraint on relative sea level in Marine Isotope Stage 3 in the Larsemann Hills, Lambert Glacier region, East Antarctica (31 366-33 228calyrBP) Quat Sci Rev 28) 26892696

Hussain S A and Saxena A (2008) Distribution and status of Antarctic seals and penguins along the Princess Astrid Coast, East Antarctica Indian J Marine Sci 37(4) 455-461

IUCN (2016) IUCN Red List -http://iucnredlist.org/ - Accessed on $15^{\text {th }}$ July 2016

Kumar R S and Johnson J A (2014) Aerial surveys for pack-ice seals along the Ingrid Christensen and Princess Astrid Coasts, East Antarctica J Threat Taxa 6(9) 6230-6238

McMahon C R and Burton H R (2005) Climate change and seal survival: evidence for environmentally mediated changes in elephant seal, Mirounga leonina, pup survival Proc $R$ Soc Lond B Biol Sci 272 923-928

Mehlum F, Gjessing Y, Haftorn S and Bech C (1988) Census of breeding Antarctic Petrels Thalassoicaantarctica and physical features of the breeding colony at Svarthamaren ,Dronning Maud Land, with notes on breeding Snow Petrels Pagodroma nivea and South Polar Skuas Catharacta maccormicki Polar Res 6 1-9

Pande A, Sivakumar K, Sathyakumar S and Mathur V B (2014) Long-term monitoring of wildlife and their habitats in Antarctica: Vertebrate surveys near Indian research stations, Unpublished Technical report submitted to National Centre for Antarctic and Ocean Research for the 33rd Indian Scientific Expedition to Antarctica, Wildlife Institute of India, Dehradun, pp. 21
Pande A, Sivakumar K, Sathyakumar S, Mondol S and Mathur V B (2015) Sentinels of south: seabird monitoring in southern ocean and Antarctica, Conference presentation at XV Annual Research Seminar, 1- 4 Sept, 2015, Wildlife Institute of India, Dehradun

Ribic C A, Chapman E and Fraser W R (2008) Top predators in relation to bathymetry, ice, and krill during austral winter in Marguerite Bay, Antarctica Deep-Sea Res II 55 485-499

Robbins J, Dalla Rosa L, Allen J M et al. (2011) Return movement of a humpback whale between the Antarctic Peninsula and American Samoa: A seasonal migration record Endanger Species Res 13 117-121

Sathyakumar S (1995) Developing a long-term monitoring programme for birds and mammals in the Indian Ocean and Antarctica using GPS and GIS technologies, Technical Publication, Department of Ocean Development 12 207219

Siegel V, Reiss C S, Dietrich K S et al. (2013) Distribution and abundance of Antarctic krill (Euphausiasuperba) along the Antarctic Peninsula Deep Sea Res I 77 63-74

Singh S M, Ochyra R, Pednekar S M et al. (2012) A Holocene moss species preserved in lake sediment core and the present moss diversity at Schirmacher Oasis, Antarctica Antarct Sci 24 353-358

Sivakumar K and Sathyakumar S (2012) Climate Change and its impact on the distribution of birds in Southern Indian Ocean and Antarctica. In: G. K. Saha (Ed) Climate Change: Man and Environment. Daya Publishers, Delhi. pp. 300

Smith R C, Ainley D, Kaber K et al. (1999) Marine ecosystem sensitivity to historical climate change in the Antarctic Peninsula Bio Science 49 393-404

Sydeman W J, Poloczanska E, Reed T E and Thompson S A (2015) Climate change and marine vertebrates Science 350 $772-777$

Tasker M L, Jones P H, Dixon T and Blake B F (1984) Counting seabirds at sea from ships: A review of methods employed and a suggestion for a standardized approach The Auk $\mathbf{1 0 1}$ $567-577$

Trathan P N, García Borboroglu P, Boersma D et al. (2015) Pollution, habitat loss, fishing, and climate change as critical threats to penguins Conserv Biol 29 31-41

Walther G R, Post E, Convey Pet al. (2002) Ecological responses to recent climate change Nature 416 389-395

Weimerskirch H, Inchausti P, Guinet C and Barbraud C (2003) Trends in bird and seal populations as indicators of a system shift in the Southern Ocean Antarct Sci 15 249256 